\title{
Erythema multiforme in the esophagus
}

\author{
J. Vandewalle'1,3, P. Van Eyken², M. Struyve ${ }^{1}$ \\ (1) Department of Gastroenterology, Ziekenhuis Oost-Limburg (ZOL), Genk, Belgium ; (2) Department of Pathology, Ziekenhuis Oost-Limburg (ZOL), Genk, Belgium ; \\ (3) Department of Internal Medicine, University Hospitals Gasthuisberg, Leuven, Belgium.
}

\begin{abstract}
Erythema multiforme is an immune-mediated mucocutaneous disorder. Mucosal involvement usually affects the oral region, the genitals or the eyes. We report a case of esophagitis caused by erythema multiforme in a patient diagnosed with lung cancer. Esophageal manifestation in erythema multiforme is rarely seen. Besides esophagitis it can lead to esophageal strictures. Erythema multiforme is mostly triggered by infection or drugs but the association with malignancy has been described. (Acta gastroenterol. belg., 2021, 84, 513-515).
\end{abstract}

Key words: dysphagia, esophagitis, esophageal stricture, erythema multiforme, malignancy.

\section{Introduction}

Erythema multiforme (EM) is an immune-mediated mucocutaneous disorder characterised by erythematous target lesions on the skin, sometimes accompanied by oral, genital or ocular mucosal bullae or erosions. EM with severe mucosal involvement is defined as erythema multiforme major. Infrequently, EM presents with only mucosal involvement (1). EM is triggered by infections (mostly herpes simplex virus (HSV) or Mycoplasma pneumoniae) and drugs (especially nonsteroidal antiinflammatory drugs, antibiotics or antiepileptics). Rarely, EM is associated with malignancies and autoimmune diseases like inflammatory bowel disease. Although EM is usually self-limited, frequent episodes over the course of years can lead to recurrent disease. Esophageal involvement and complications in EM, resulting in esophagitis and esophageal strictures respectively, have been described (2).

\section{Case}

A 61-year old man was referred to the gastroenterology department with complaints of dysphagia for solid food and odynophagia for one year. The patient had an extensive medical history of diabetes mellitus type 2, chronic kidney disease (stage 4), ischaemic cardiomyopathy and peripheral vascular disease. Four years ago he experienced similar complaints of dysphagia and was diagnosed with esophagitis on esophagogastroduodenoscopy (EGD). Biopsies from the distal part of the esophagus showed

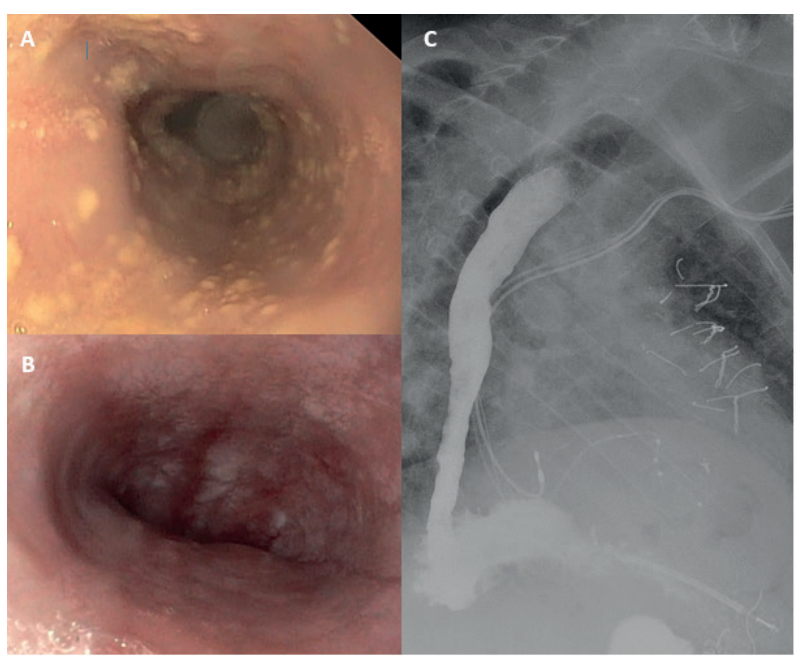

Figure 1. - EGD image showing white plaques adherent to the mucosa of the esophagus (1A). Multiple furrows and concentric rings in the distal two thirds of the esophagus (1B). Stasis of fluid substances on barium esophagram (1C).

characteristics of both reflux and possible manifestation of EM. Proton pump inhibitors (PPIs) were initiated in high dose (Pantoprazole $40 \mathrm{mg}$ twice daily) by which symptoms disappeared. Control EGD three months later showed remaining gastroesophageal reflux disease (GERD) wherefore PPIs were continued.

At present, an esophageal manometry showed aperistalsis in the distal two thirds of the esophagus. Barium esophagram revealed stasis of contrast both for solid and fluid substances without evidence for any motility disorders (figure 1C). A new EGD was performed and demonstrated a tortuous distal part of the esophagus with macroscopic signs of candida esophagitis and reflux esophagitis grade B according to the Los Angeles classification (figure 1A) despite the use of PPIs in high dose for over three years. Biopsies were taken from the distal part of the esophagus and the stomach. The histopathologic findings of the biopsy specimen from the esophagus were again compatible with EM (figure 2A). Immunohistochemical staining for herpes virus was negative. PAS (periodic acid-Schiff) staining showed no pseudohyphae. The patient had no history of

Correspondence to : J. Vandewalle, Schiepse bos 6, 3600 Genk, Belgium.

E-mail : vandewalle.jan@student.kuleuven.be

Submission date : 29/07/2020

Acceptance date : 14/10/2020 


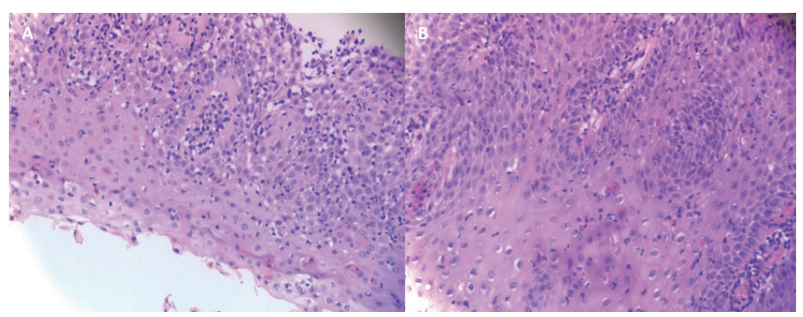

Figure 2. - Esophageal biopsies from our patient. The esophageal mucosa shows marked intra-epithelial lymphocytosis and individual squamous cell necrosis, suggestive for erythema multiforme.

skin disorders. Clinical examination by the dermatology department revealed no mucosal or cutaneous lesions. A course of Fluconazole $200 \mathrm{mg}$ each day during 14 days brought temporary relief but symptoms returned once therapy was stopped. A new EGD four months later showed furrows and multiple concentric rings in the distal third part of the esophagus (figure 1B). New esophageal biopsies confirmed the presence of EM (figure 2B). Around the same time, the patient was diagnosed with small cell lung cancer (SCLC) and sternal metastasis. Metoclopramide $10 \mathrm{mg}$ three times daily was started for diabetic gastroparesis. His symptoms improved slightly. After association of Pyridostigmine $10 \mathrm{mg}$ three times daily, all complaints disappeared. The patient died four months later due to his oncological condition.

\section{Discussion}

EM is a mucocutaneous disorder that typically presents with erythematous skin lesions. The frequency of mucosal involvement is estimated at $20-65 \%$ (2). Besides the mouth, the eyes and the genitals, EM rarely affects the pharynx, the upper respiratory tract or the esophagus. Infrequently, EM presents with only mucosal involvement. In a study of 48 patients with recurrent EM, five had mucosal lesions (all oral and two genital) in the absence of cutaneous damage (3). The majority of case reports describing esophageal manifestation in EM are associated with HSV - or M. pneumoniae infection $(4,5)$. However, an identifiable cause in patients with recurrent $\mathrm{EM}$ is not always found. In the absence of obvious causes, the possibility of EM esophagitis triggered by the malignancy should be considered in this case, especially since case reports of recurrent EM in patients with solid organ cancers have been described (6). Mostly, EM is diagnosed clinically and does not require further testing. Erosive esophagitis, erythematous friable mucosa and esophageal ulcerations have been reported on EGD in patients with EM in the esophagus. In a further stage, esophageal strictures are a possible complication. Based on EGD findings, GERD refractory to PPI, candidiasis and eosinophilic esophagitis should be included in the differential diagnosis. In this case, pathology was not suggestive for any of these entities. Esophageal $\mathrm{pH}$ monitoring was not performed. Esophageal motility disorders such as achalasia or systemic sclerosis were excluded on barium esophagram and manometry. In case of esophageal strictures, more common causes (caustic ingestion, nasogastric intubation and mediastinal irradiation) should be considered.

A biopsy specimen may be helpful in excluding other diagnosis. The pathologic findings in EM include necrotic keratinocytes, lymphocyte exocytosis into the epithelium and degeneration of the basal epidermal cells (1). Histologically, these findings show similarities with two other mucocutaneous disorders involving the esophagus: Stevens-Johnson syndrome (SJS) and toxic epidermal necrolysis (TEN). It is not always easy to differentiate EM from SJS and TEN. However, SJS and TEN are associated with different skin lesions and a more severe disease course with constitutional symptoms. Furthermore, SJS and TEN are practically always druginduced, unlike EM (7). Literature regarding EM should be interpreted with caution since it was considered to be on the same pathological spectrum as SJS and TEN in the past. Lastly, paraneoplastic pemphigus (PNP) also affects the esophagus and is associated with malignancy, mostly lymphoproliferative disorders (8). It is easily differentiated from EM on histology and immunofluorescence studies.

Management of EM depends on the severity. Treatment of the underlying cause is the most important. In case of severe mucosal involvement topical corticosteroids, oral antiseptics and anesthetic solutions are recommended based on expert opinion (6). Recurrent EM may be treated with Acyclovir, even if HSV is not the obvious trigger (9). Specific therapy for EM esophagitis is not described. In the case reports available, patients are most often treated with PPIs and corticosteroids. Esophageal dilatation has been performed for esophageal strictures due to EM (10).

In conclusion, EM rarely affects the esophagus and potentially leads to esophagitis or esophageal strictures. We describe the case of a patient with EM esophagitis. The association with a malignancy should be considered in this patient. To the best of our knowledge, this would be the first case of EM esophagitis caused by malignancy. In patients with dysphagia, an esophageal manifestation of mucocutaneous disorders should be included in the differential diagnosis, especially in patients with skin diseases. In case of esophageal involvement in EM, the possibility of a malignancy should be investigated when obvious triggers such as HSV or M. pneumoniae infection are absent.

\section{Conflict of Interests}

Nothing to disclose.

\section{References}

1. SOKUMBI O., WETTER D.A. Clinical features, diagnosis, and treatment of erythema multiforme: a review for the practicing dermatologist. International Journal of Dermatology, 2012, 51(8) : 889-902 
2. HUFF J.C., WESTON W.L., TONNESEN M.G. Erythema multiforme: a critical review of characteristics, diagnostic criteria, and causes. Journal of the American Academy of Dermatology, 1983, 8(6) : 763-775.

3. WETTER D.A., DAVIS M.D. Recurrent erythema multiforme: clinical characteristics, etiologic associations, and treatment in a series of 48 patients at Mayo Clinic, 2000 to 2007. Journal of the American Academy of Dermatology, 2010, 62(1) : 45-53

4. CARUCCI L.R., LEVINE M.S., RUBESIN S.E. Diffuse esophageal stricture caused by erythema multiforme major. American Journal of Roentgenology, 2003, 180(3) : 749-750.

5. SABBAH M., BIBANI N., TRAD D., OUAKAA A., ELLOUMI H., GARGOURI D. Oesophageal localisation of Erythema Multiforme due to Herpes Simplex Virus. Clin. Case Rep. Int., 2018, 2(1) : 1061.

6. TRAYES K.P., LOVE G., STUDDIFORD J.S. Erythema multiforme : recognition and management. American family physician, 2019, 100(2) : 82-88.
7. ASSIER H., BASTUJI-GARIN S., REVUZ J., ROUJEAU J.C. Erythema multiforme with mucous membrane involvement and Stevens-Johnson syndrome are clinically different disorders with distinct causes. Archives of dermatology, 1995, 131(5) : 539-543.

8. WAKAHARA M., KIYOHARA T., KUMAKIRI M., UEDA T., ISHIGURO K., FUJITA, T., et al. Paraneoplastic pemphigus with widespread mucosal involvement. Acta Derm. Venereol., 2005, 85(6) : 530-532.

9. LAMOREUX M.R., STERNBACH M.R., HSU W.T. Erythema multiforme. American family physician, 2006, 74(11) : 1883-1888.

10. VIARNAUD A., INGEN-HOUSZ-ORO S., MARQUE M., VALEYRIEALLANORE L., ORTONNE N., GUEUDRY J., et al. Severe sequelae of erythema multiforme: three cases. Journal of the European Academy of Dermatology and Venereology, 2018, 32(1) : 34 . 